\title{
Dynamic patterns of postural fluctuations during quiet standing: a
}

\section{recurrence quantification approach}

\author{
Hamed Ghomashchi ${ }^{1, *}$, Ali Esteki ${ }^{2}$, Ali Motie Nasrabadi ${ }^{3}$, Julien Clinton Sprott ${ }^{4}$, Farid \\ BahrPeyma $^{5}$
}

${ }^{1}$ Department of Mechanical Engineering, Faculty of Mechanical and Industrial Engineering, Islamic Azad University, Qazvin Branch, Qazvin, Iran

E-mail: ghomashchi@qiau.ac.ir

${ }^{2}$ Department of Biomedical Engineering, Faculty of Medicine, Medical Campus, Shahid Beheshti University, Tehran, Iran

E-mail: aesteki@sbmu.ac.ir

${ }^{3}$ Department of Biomedical Engineering, Faculty of Engineering, Shahed University, Tehran, Iran

E-mail: nasrabadi@shahed.ac.ir

${ }^{4}$ Department of Physics, University of Wisconsin, Madison, WI 53706 USA

E-mail: csprott@wisc.edu

${ }^{5}$ Department of Physical Therapy, Faculty of Medicine, Tarbiat Modares University, Tehran, Iran

E-mail: bahrpeyf@modares.ac.ir

*Corresponding Author:

Hamed Gomashchi, Room 419, Department of Mechanical Engineering, Faculty of Mechanical and Industrial Engineering, Islamic Azad University, Qazvin Branch, Pajooheshgaran Blvd., Nokhbegan Blvd., Daneshgah Ave. Barajin, Qazvin, Iran

Telephone: +989126073741

Fax: +98 02813670051

E-mail: ghomashchi@qiau.ac.ir and h_ghomashchi@yahoo.com 


\begin{abstract}
During quiet standing the human body continuously moves about an upright posture in an erratic fashion. Conventional posturographic analyses that ignore structure of postural steadiness time series do not fully characterize properties of sway dynamics. Recurrence quantification analysis is a technique that can extract the dynamics of postural fluctuations through several variables. In this study standing still sway dynamics of intact and deteriorated postural control systems were investigated by recurrence quantification of stabilograms. The results indicated that both normal and changed postural fluctuations time series, despite erratic and irregular appearance, contain a hidden structure. Although the two components of postural sway originate from an integrated control system, they exhibit distinct dynamical patterns. More determinism, greater stability and higher degrees of non stationarity were observed in foreaft movements. Our findings reveal that decay of postural control mechanism affects dynamical properties of postural control system (especially along mediolateral direction because of the type of the impairment). Determinism, non stationarity and rigidity of balance program are increased due to deterioration of postural control system. These findings imply that these measures not only can be used as the pathologic measures to discriminate between groups differences but also provide new openings to understand the nature postural sway.
\end{abstract}

\title{
Running Title
}

Recurrence quantification of body sway

\section{Keywords}

Postural control, Quiet standing, Balance impairment, Nonlinear Dynamics, RQA 


\section{Introduction}

Balance control during quiet standing is one of the essential activities that human learn in childhood and perform at subconscious level. Despite its apparent simplicity, the task of maintaining an upright posture involves a complex sensorimotor control system. Various mechanisms and neurophysiologic sensory systems including visual, vestibular, and somatosensory systems contribute to our stability during quiet standing and respond to internal or external perturbations [Shumway-Cook \& Woollacott, 2006].

The complex behavior of standing still postural control mechanism has been studied using different mathematical linear and nonlinear quantifiers. Many researchers have used linear posturographic measures and summary statistics of $\mathrm{COP}^{1}$ time series, which by definition ignore temporal structure of time series, to characterize postural sway during quiet standing and to study the influences of different factors on postural steadiness [Nichols et al., 1995], [DeHaart et al., 2004], [Norris et al., 2005], [Raymakers et al., 2005], [Blaszczyk et al., 2007], [Esteki et al., 2009]. Linear measures such as path length or sway range can be used to describe how much the COP moves around (quantity of movement), but these measures do not give any information about how well coordinated the movement is (quality of movement) and provide no means to underlying control structure, so dynamical system analysis seems to be useful in this regard. The complex and unpredictable behavior exhibited by sensorimotor control system, as illustrated in Fig.1, has been examined from different perspectives. Many literatures suggested that this complexity may be instances of deterministic physiological chaos and arises from a low dimensional chaotic system [Newell et al. 1993], [Yamada, 1995], [Pascolo et al., 2005], [Roerdink et al. 2006], [Donker et al. 2007], [Ladislao \& Fioretti, 2007] while some others believe that is the blend of deterministic and random processes [Collins \& Luca, 1993], [Newell et al. 1997], [Duarte \& Zatsiorsky, 2000], [Amoud et al., 2007].

*** FIGURE 1 NEAR HERE ***

\footnotetext{
${ }^{1}$ Center of pressure
} 
Standing posture is still poorly understood and weakness of postural control mechanism certainly plays a role in balance control during quiet standing. Knowledge of sway dynamics can provide new insights into nature of disease process and rehabilitation effects that may be unattainable using the measures that ignore the time varying properties of sway.

In our previous study we investigated the existence of low dimensional chaos within sway dynamics of normal and deteriorated standing still postural control mechanisms. We could not find any evidence to document existence of low dimensional attractors in COP dynamics of neither normal nor deteriorated postural control mechanisms. We never disprove the possibility of existence of chaos within standing still sway dynamics but our findings indicated that none of them may not be modeled as a low dimensional chaotic process and if there is any chaos within dynamics of quiet standing postural fluctuations it might be of a dimension too high to detect [Ghomashchi et al. 2010].

In this study we used Recurrence Quantification Analysis (RQA) to evaluate dynamical properties of postural fluctuations of normal and deteriorated postural control mechanisms during quiet standing. RQA can extract a system's dynamics by quantifying the system's repeatability through several variables. It is shown that RQA is sensitive to changes in postural sway dynamics in response to variations in availability of sensory information used to control balance and optical flow [Riley et al. 1999], to balance expertise [Schmit et al. 2005], to balance impairments [Schmit et al. 2006], and also embedding parameters [Hasson et al. 2007].

\section{Methods}

\subsection{Participants and Procedures}

In this study, to extract the dynamics of normal and deteriorated postural control systems, two distinctly different groups were considered. Postural control system of healthy young adults is considered as the system with normal dynamics and of elderly stroke patients with severe balance disorders is considered as the abnormal dynamical system. 32 stroke patients (17 male and 15 female) with a first hemispheric 
intracerebral infarction or hematoma with less than one year $(6.9 \pm 4.2$ months) post stroke time with the age of $60.59 \pm 8.64$ years old and $\mathrm{BMI}^{1}$ index of $25.01 \pm 4.51$ and 29 healthy young adults $(16$ male and 13 female) with the age of $25.90 \pm 3.32$ years old and $23.54 \pm 2.88$ BMI index, without known motor impairments or movement-related disorders, participated in the experiment. There was no significant difference between BMI indexes of two groups.

Postural fluctuations were evaluated using a dynamic dual force platform (SOT\#1, EquiTest testing system, NeuroCom International Inc., Clackamas, OR). The system was equipped with a movable visual surround and support surface that could rotate in the $\mathrm{AP}^{2}$ plane. Two $22.9 \times 45.7 \mathrm{~cm}$ force plates connected by a pin joint were used to collect COP coordinates at $100 \mathrm{~Hz}$.

Participants were instructed to stand in an upright posture in a standardized foot placement on the platform based on each subject's height according to the manufacturer's protocol [NeuroCom Internatinal, Inc., 2001]. Participants stood barefoot with their arms relaxed at their sides, their eyes open and look straight ahead fixed on a point in front of them. They were instructed to concentrate on their stability, stand freely, and have no other mental tasks. Each participant performed a set of 3 trials each lasting 20 seconds.

\subsection{Data Processing}

Prior to all analyses to remove stationarity effects, mean and linear trend of the signals were removed and the signals were variance normalized. Spectral analysis was performed and the signals were processed by a $2^{\text {nd }}$ order low pass Butterworth filter with cutoff frequency of $15 \mathrm{~Hz}$.

\subsection{RQA Input Parameters}

RQA requires reconstruction of a dynamical system's state space from a single scalar time series, $x(t)$, through the process of embedding the time series in m-dimensional phase space by delayed vectors as $\mathrm{x}\left(\mathrm{t}_{\mathrm{i}}\right), \mathrm{x}\left(\mathrm{t}_{\mathrm{i}}+\tau\right), \mathrm{x}\left(\mathrm{t}_{\mathrm{i}}+2 \tau\right), \ldots$.

\footnotetext{
${ }^{1}$ Body mass index

${ }^{2}$ Anterior- posterior
} 
In order to reconstruct the state space of a dynamical system, two problems will need to be solved. The first concerns how to select the time delay $(\tau)$. With very small delays, the resulting delayed vectors will be nearly the same, and so the trajectories in the embedding space will all be compressed into a long thin volume equivalent to a diagonal line in the state space. On the other hand, a large delay may produce coordinates which are essentially unrelated. For nonlinear systems one of the favored approaches is choosing the delay coincides with the first local minimum of auto mutual information function that maximizes independence between state vectors. This value was not repeatable in COP time series of neither normal nor deteriorated postural fluctuations but it can be characterized by a mean value \pm standard deviation in each group of COP time series. So, the average of first local minimums of auto mutual information functions of COP time series is considered as the proper time delay. The second problem concerns how to determine the embedding dimension (d) of the system. Our approach to this problem was to use an analytic method known as False Nearest Neighbors (FNN) algorithm proposed by Kennel et al. [1992]. Embedding dimension is chosen when the percentage of false nearest neighbors as a function of the embedding dimension drops or closest to zero.

A constant radius (r) of $2.5 \%$ average of mean distances between data points in reconstructed state space was chosen based on radius selection method proposed by Riely et al. [1999] and conservative choice of 2 points was considered as the number of successive points defining a line segment in recurrence plots.

Calculation of first local minimums of auto mutual information functions and mean distances between data points in embedding space were performed in MATLAB ${ }^{\circledR}$ (Matworks ,Inc.) and to calculate embedding dimension of the time series Chaos Data Analyzer (CDA) software package (Physics Academic Software, by J.C. Sprott and G. Rowlands) was used which follows FNN algorithm [Sprott \& Rowlands, 2003]. RQA software package [Webber, 2009] was used to compute dynamical properties of postural sway. Detailed description of RQA output parameters can be found in Riely et al. [1999] and Riley \& Van Orden [2005]. 


\subsection{Statistical Analyses}

All statistical analyses were performed using SPSS software package version 11.5 (SPSS Inc., Chicago, IL, USA). Normality of the results were checked, independent samples $\mathrm{T}$ tests with $95 \%$ level of confidence were used to evaluate between groups differences and paired samples $\mathrm{T}$ tests with $95 \%$ level of confidence were used to evaluate statistical differences between $\mathrm{AP}$ and $\mathrm{ML}^{1}$ variables in each group.

\section{Results}

The results of calculating RQA input parameters are listed in table 1. Since the differences between input parameters' values affect RQA measures, constant time delay of 11 data points ( average of first local minimums of auto mutual information functions), constant embedding dimension of 2 and constant radius of $0.04(2.5 \%$ average of mean distances $)$ were used in the analysis.

\section{*** TABLE 1 NEAR HERE ***}

Determinism, maximum diagonal line length, information entropy and trend of each group of COP time series were calculated from recurrence plots (Fig.2) and the results are shown in Figs. 3-6. Since there was a large number of COP time series, in this study we have focused on quantitative measures derived from recurrence plots rather than qualitative features from texture analysis.

*** FIGURE 2 NEAR HERE ***

Marked differences were found between dynamical properties of normal and deteriorated postural fluctuations and also between AP and ML components of COP time series in each group.

\footnotetext{
${ }^{1}$ Mediolateral
} 
*** FIGURE 3 NEAR HERE ***

*** FIGURE 4 NEAR HERE ***

The results indicate that in both groups the determinism as well as the information entropy of postural fluctuations along AP direction is significantly higher than ML direction. In normal postural control system the trend and the maximum diagonal line length along AP direction are significantly greater than ML counterparts.

*** FIGURE 5 NEAR HERE ***

*** FIGURE 6 NEAR HERE ***

Postural fluctuations of deteriorated control mechanism are more deterministic than normal one, especially along ML direction. The similar, information entropy of postural steadiness time series of deteriorated postural control system, particularly ML, is higher than normal control system. Maximum diagonal line lengths of both AP and ML COP time series of changed control system are greater than intact postural control system, whereas only the trend along ML direction is significantly different between two groups.

\section{Discussion}

Analyzing of COP time series of normal postural control system reveals that although the two components of COP trajectories are the outputs of an integrated control system, they have different appearances. The determinism shows that postural fluctuations along AP direction are more repeatable or more structured than ML. Since determinism can readily discriminate between directions differences, information entropy which defines the complexity of deterministic structure may not give us additional 
useful information. In normal control system in addition to repeatability (and/or complexity) other dynamical characteristics of postural control system (i.e. the maximum diagonal line length and the trend) are significantly different along AP and ML directions. The length of largest diagonal line, which inversely is related with largest Lyapunov exponent, is a measure local dynamic instability and the less line length indicates more chaos in the system. Since there is no chaos (at least low dimensional) within dynamics of postural sway, this result should be interpreted in this way: the nearby trajectories of embedded ML COP time series diverged more rapidly comparing to embedded AP COP time series that leads to less determinism along ML direction. The trend is a measure of the degree of non stationarity of embedded COP time series and ML COP time series are more stationary than AP counterparts in normal postural control system.

It is generally believed that in case of postural control impairments both AP and ML postural movments are changed in a same manner and this may be the reason that many researchers concentrated only on a component of COP trajectories (especially AP) to study postural control behavior during quiet standing [Nichols et al., 1995], [Ladislao \& Fioretti, 2007], [Esteki et al., 2009] but in this study we found different results. Degradation of postural control system causes that the differences between dynamical characteristics (except repeatability) of AP and ML COP time series be lost. Our findings also shown that there are no significant differences between dynamical characteristics of normal and changed postural control systems along AP direction (except maximum diagonal line length) but they are all significantly different along ML direction. It implies that the neurological impairment (hemiplegia) more affected on ML direction and significantly increased the determinism, local stability as well as non stationarity of ML movements and made them comparable with AP counterparts. Due to the reduced ability of symmetrical weight distribution between load bearing limbs, side to side load shifting causes more repeatable (more deterministic) ML sway patterns, similar to AP ones, for the patients. Lower ability of loading the paretic side and load shifting from non paretic to paretic side provide greater non stationarity for postural fluctuations along ML direction. However local instability and somehow the determinism along AP 
direction were also sensitive enough to distinguish intact and deteriorated postural control systems. But it should be noted that if the trajectory is to remain in a bounded region in the state space, the length of largest diagonal line quantifies how long two nearby trajectories stay close together. So, it is not equivalent to clinical measures of postural stability and it is better to say that it quantifies the rigidity (or flexibility) of balance programs.

These results of this study are well consistent with the results of many other literatures which indicate loss of complexity in elderlies, and in neurological and physiological diseases [Kaplan et al. 1991], [Lipsitz \& Goldberger, 1992], [Pincus \& Goldberger, 1994], [Lipsitz, 2004], [Kunhimangalam et al. 2007] because according to definitions, loss of complexity is consistent with increasing of regularity or determinism. This makes sense; in deteriorated postural control system, less complex (more deterministic) and more rigid programs are utilized to maintain balance during quiet standing.

\section{Conclusion}

In this study we investigated sway dynamics of normal and abnormal postural control mechanisms using Recurrence Quantification Analysis method. Appropriate parameter selection methods from mathematical theory of nonlinear dynamical systems are utilized to select suitable input parameters. RQA measures not only can be used as the pathological discriminating criteria for balance disorders, but also they provide useful information about underlying control mechanism and its hidden dynamics. It is important to recognize that there are orderly patterns within these outwardly unorganized and noisy looking fluctuations which are the outcomes of distinguishable different postural control programs. The results justify the inclusion of RQA quantifiers in clinical assessment of postural fluctuations to understand the disease process and the effects of rehabilitation on patients who receive physical therapy interventions. 


\section{References}

Amoud, H., M. Abadi, et al. (2007). "Fractal time series analysis of postural stability in elderly and control subjects." Journal of neuroengineering and rehabilitation $\mathbf{4}(12)$.

Blaszczyk, J. W., R. Orawiec, et al. (2007). "Assessment of postural instability in patients with parkinson disease." Experimental Brain Research 183: 107-114.

Collins, J. J. and C. J. D. Luca (1993). "Open loop and closed loop control of posture: a random walk analysis of center of pressure trajectories." Experimental Brain Research 95: 308-318.

DeHaart, M., A. C. Geurts, et al. (2004). "Recovery of standing balance in postacute stroke patients: A rehabilitation cohort study." Arch Phys Med Rehabil 85: 886-895.

Donker, S. F., M. Roerdink, et al. (2007). "Regularity of center-of-pressure trajectories depends on the amount of attention invested in postural control." Experimental Brain Research 181: 1-11.

Duarte, M. and V. M. Zatsiorsky (2000). "On the fractal properties of natural human standing." Neuroscience Letters 283: 173-176.

Esteki, A., H. Ghomashchi, et al. (2009). Can linear posturographic measures discriminate healthy subject from CVA patient during quiet standing? 15th Iranian Conference on Biomedical Engineering, Mashhad, Iran.

Ghomashchi, H., A. Esteki, et al. (2010). "Identification of dynamic patterns of body sway during quiet standing: Is it a nonlinear process?" International Journal of Bifurcation and Chaos In Press. 
Hasson, C. J., R. E. A. V. Emmerik, et al. (2007). "Influence of embedding parameters and noise in center of pressure recurrence quantification analysis." Gait \& Posture doi:10.1016/j.gaitpost.2007.05.010.

Kaplan, D. T., M. I. Furman, et al. (1991). "Aging and the complexity of cardiovascular dynamics." Journal of Biophysics 59: 945-949.

Kennel, M. B., R. Brown, et al. (1992). "Determining embedding dimension for phase space reconstruction using a geometrical construction." Physical Review A 45(6): 3403-3411.

Kunhimangalam, R., P. K. Joseph, et al. (2007). "Nonlinear analysis of EEG signals: Surrogate data analysis." ITBM-RBM doi:10.1016/j.rbmret.2007.09.006.

Ladislao, L. and S. Fioretti (2007). "Nonlinear analysis of posturographic data." $\underline{\text { Med Bio Eng Comput }}$ 45: 679-688.

Lipsitz, L. A. (2004). "Physiological complexity, aging, and the path to frailty " Sci. Aging Knowl. Environ 2004(16): pe16.

Lipsitz, L. A. and A. L. Goldberger (1992). "Loss of complexity and aging. Potential applications of fractals and chaos theory to senescence." The Journal of the American Medical Association (JAMA) 267(13): 1806-1809.

NeuroCom Internatinal, Inc. (2001). EquiTest System Version 8.0, Operator's Manual. Clackamas, Oregon. 
Newell, K. M., R. E. A. V. Emmerik, et al. (1993). "On postural stability and variability." Gait \& Posture 4: 225-230.

Newell, K. M., S. M. Slobounov, et al. (1997). "Stochastic processes in postural center-of-pressure profiles." Experimental Brain Research 113: 158-164.

Nichols, D. S., T. M. Glenn, et al. (1995). "Changes in mean center of balance during balance testing in young adults." Physical Therapy 75(8): 699-706.

Norris, J. A., A. P. Marsh, et al. (2005). "Ability of static and statistical mechanics posturographic measures to distinguish between age and fall risk." Journal of Biomechanics 38: 1263-1272.

Pascolo, P. B., A. Marini, et al. (2005). "Posture as a chaotic system and an application to the Parkinsons disease." Chaos, Solitons and Fractals 24: 1343-1346.

Pincus, S. M. and A. L. Goldberger (1994). "Physiological time series analysis: What does regularity quantify?" American Journal of Physiology 266 (Heart Circulatory Physiology 35): H1643_H1656.

Raymakers, J. A., M. M. Samson, et al. (2005). "The assessment of body sway and the choice of the stability parameter(s)." Gait \& posture 21: 48-58.

Riley, M. A., R. Balasubramaniam, et al. (1999). "Recurrence quantification analysis of postural flactuation." Gait \& Posture 9: 65-78. 
Riley, M. A. and G. C. VanOrden (2005). Tutorials in contemporary nonlinear methods for the behavioral sciences, Retrieved March 1, 2005, from http://www.nsf.gov/sbe/bcs/pac/nmbs/nmbs.jsp.

Roerdink, M., M. DeHaart, et al. (2006). "Dynamical structure of center-of-pressure trajectories in patients recovering from stroke." Experimental Brain Research 174: 256-269.

Schmit, J. M., D. I. Regis, et al. (2005). "Dynamic patterns of postural sway in ballet dancers and track athletes." 163: 370-378.

Schmit, J. M., M. A. Riley, et al. (2006). "Deterministic center of pressure patterns characterize postural instability in Parkinson's disease." Experimental Brain Research 168: 357-367.

Shumway-Cook, A. and M. H. Woollacott (2006). Motor control: Translating research into clinical practice, Lippincott Williams \& Wilkins.

Sprott, J. C. and G. Rowlands (2003). Chaos Data Analyzer User's Manual. Raleigh, North carolina, Physics Academic Software.

Webber, C.L., Jr. (2009). Introduction to recurrence quantification analysis. RQA version 13.1 README.PDF: http://homepages.luc.edu/ cwebber/

Yamada, N. (1995). "Chaotic swaying of upright posture." Human movement science 14: 711-726. 


\section{Captions}

Figure 1. Left panel: a typical 20 seconds COP trajectory where $\mathrm{x}$ and $\mathrm{y}$ respectively correspond to mediolateral and anterior posterior directions. Right panel: corresponding time series.

Figure 2. $\quad$ Recurrence plot of a sample ML COP time series

Figure 3. $\%$ Determinism of embedded AP and ML COP time series of both normal and deteriorated postural control systems.

Figure 4. Maximum diagonal line lengths of embedded AP and ML COP time series of normal and deteriorated postural control systems.

Figure 5. Entropy of embedded AP and ML COP time series of normal and deteriorated postural control systems.

Figure 6. Trends of embedded AP and ML COP time series of normal and deteriorated postural control systems.

Table 1. Descriptive statistics of first local minimums of auto mutual information functions of COP time series, embedding dimensions and mean distances between data points in reconstructed space. 

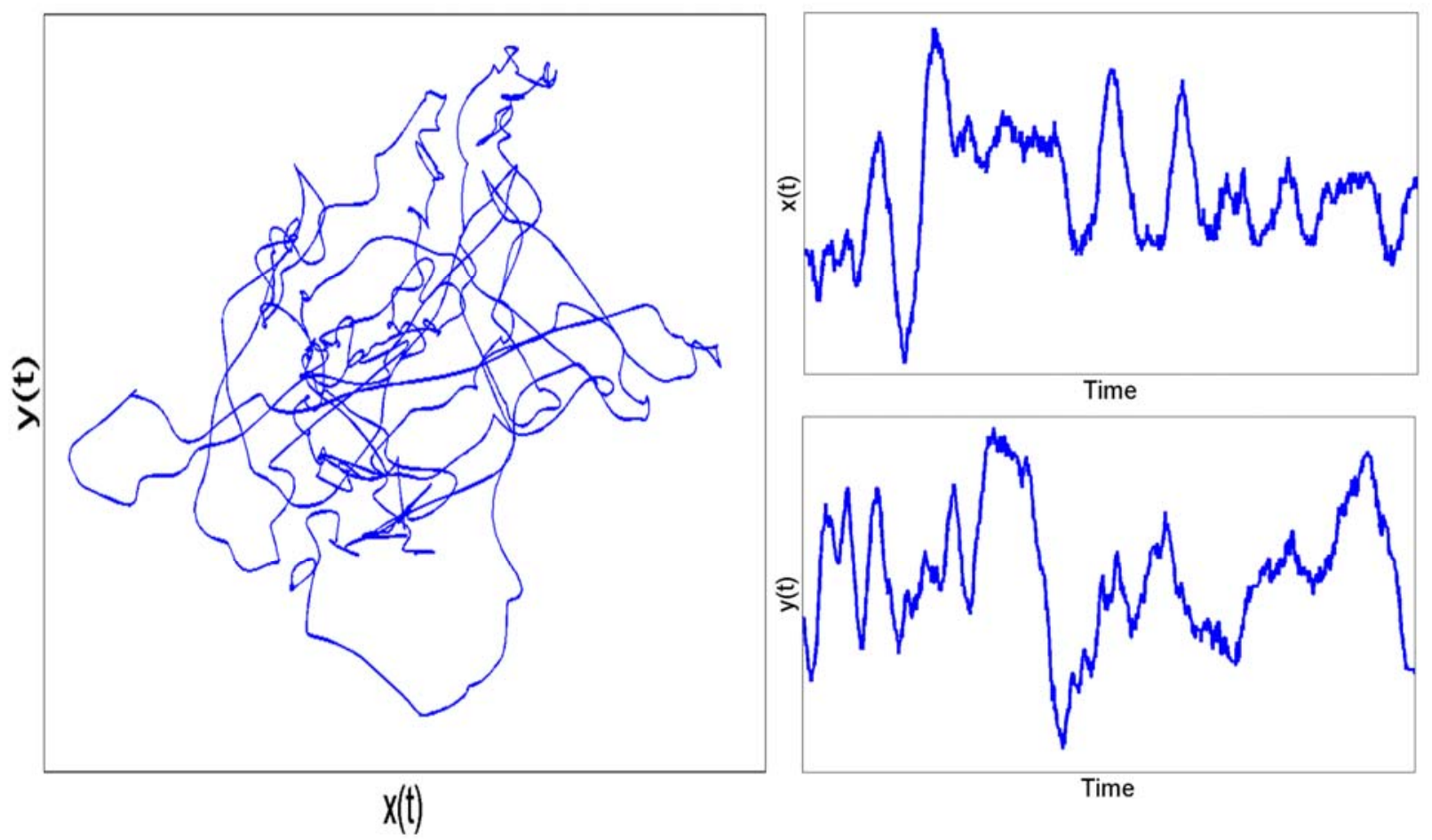

Fig. 1. Left panel: a typical 20 seconds COP trajectory where $x$ and $y$ respectively correspond to mediolateral and anterior posterior directions. Right panel: corresponding time series.

\section{(FIGURE 1)}




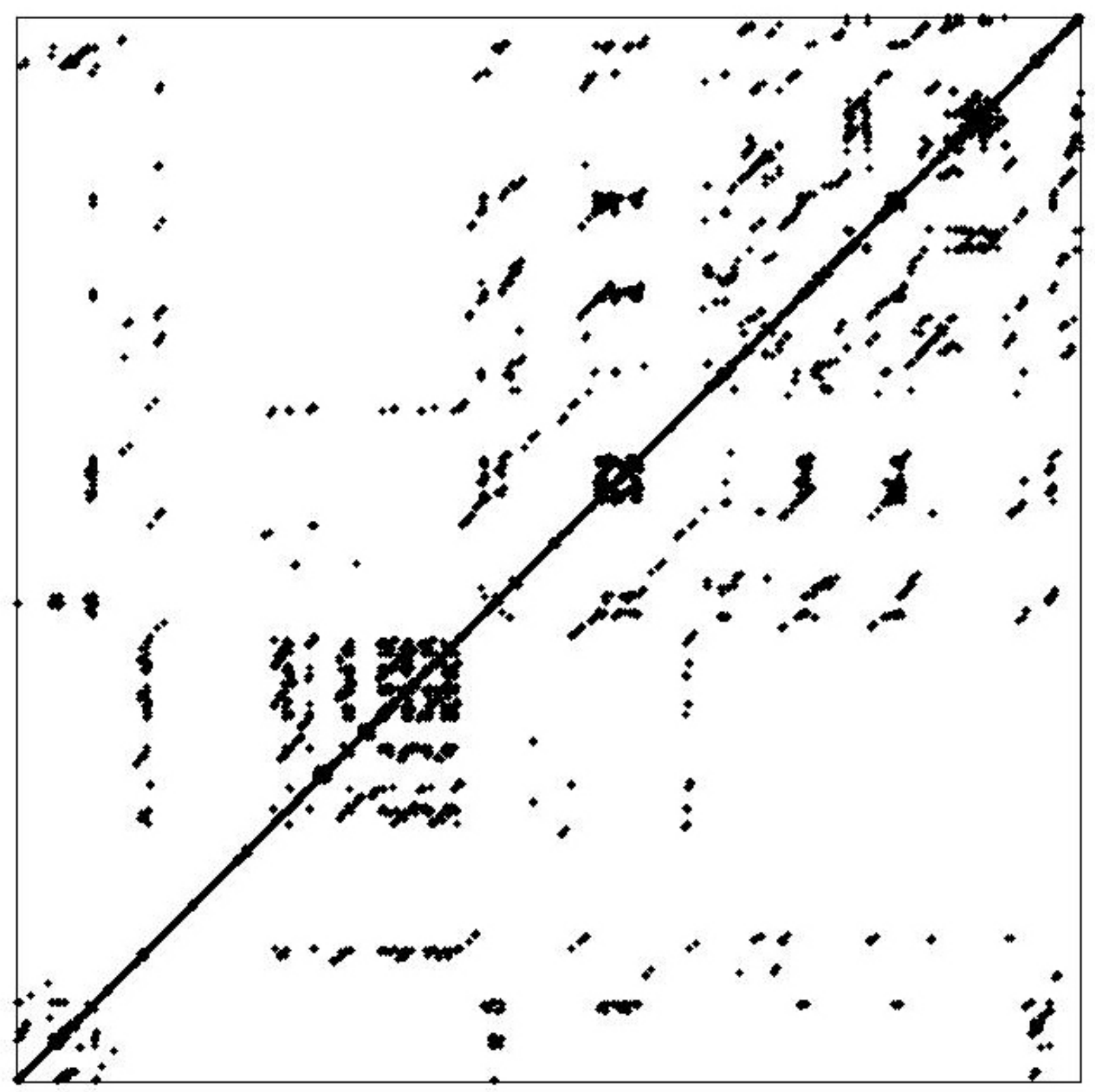

Fig. 2. Recurrence plot of a sample ML COP time series

(FIGURE 2) 


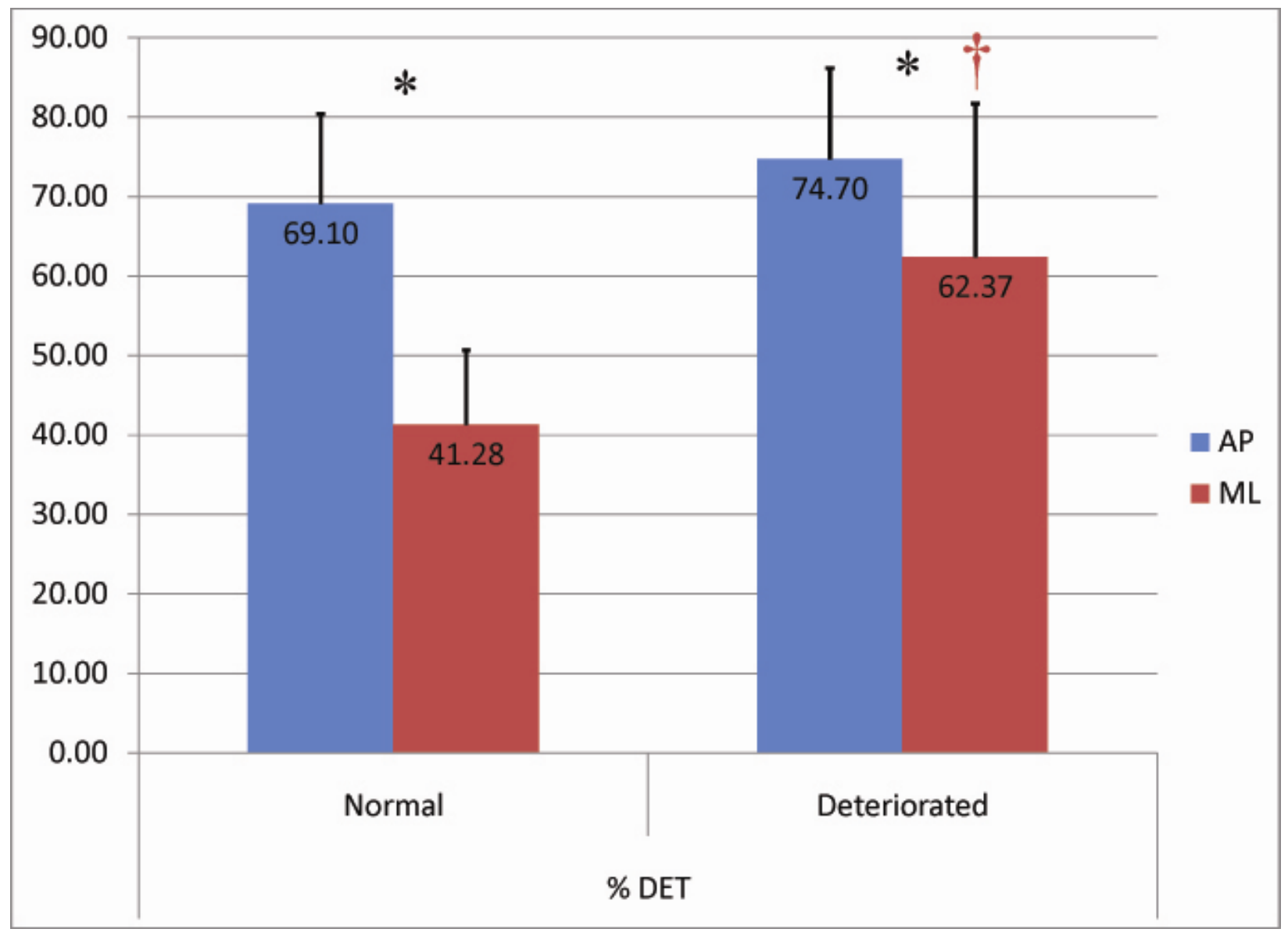

Fig 3. \% Determinism of embedded AP and ML COP time series of both normal and deteriorated postural control systems.

$\dagger$ : Deteriorated postural control system is significantly different from normal one along ML direction $\left(p_{\mathrm{AP}}=0.097, \mathrm{p}_{\mathrm{ML}}=\mathbf{0 . 0 0 0}\right)$.

*: AP is significantly different from ML in both groups $\left(p_{\text {Normal }}=\mathbf{0 . 0 0 0}, p_{\text {Deteriorated }}=\mathbf{0 . 0 0 1}\right)$.

\section{(FIGURE 3)}




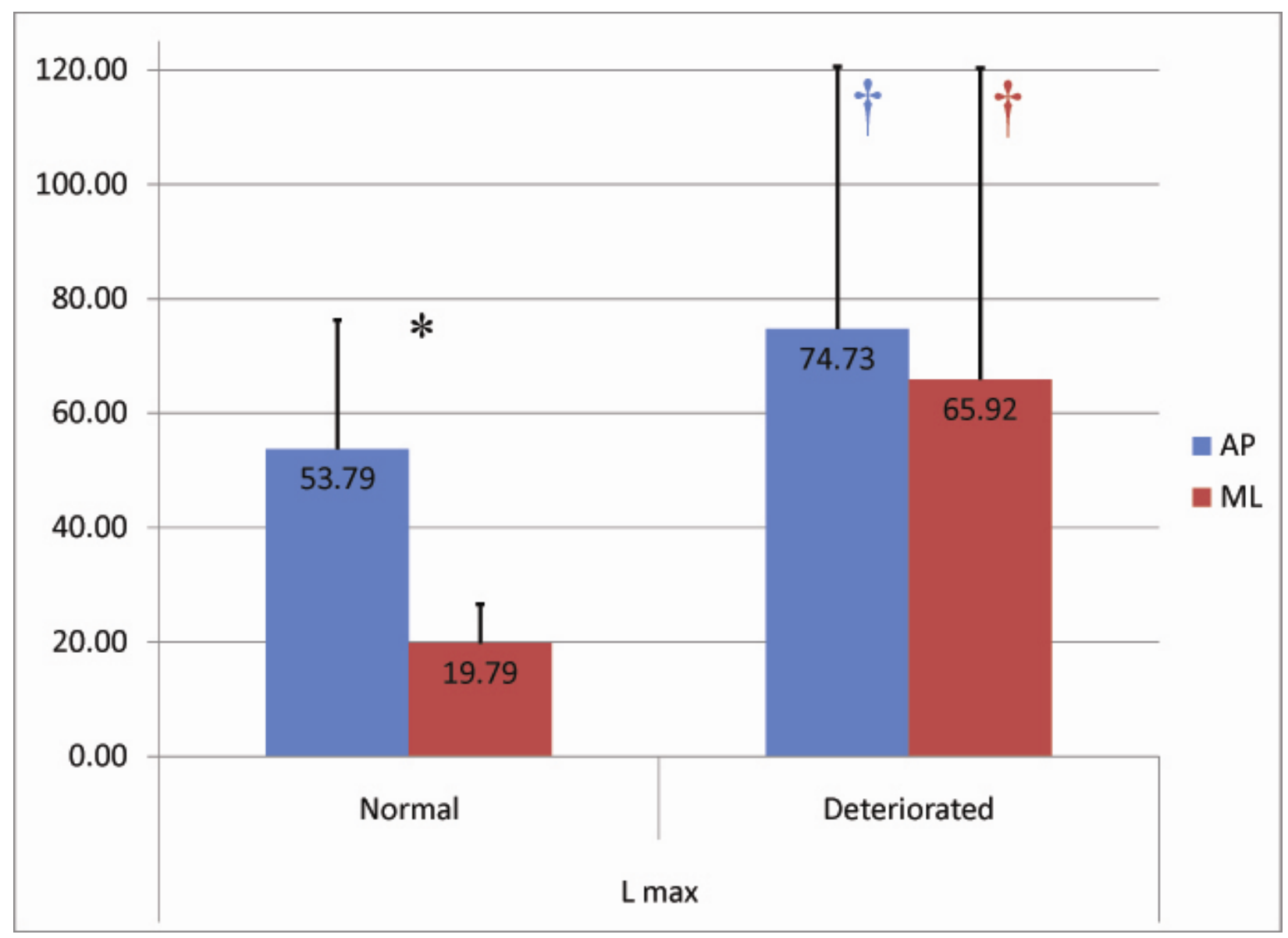

Fig 4. Maximum diagonal line lengths of embedded AP and ML COP time series of normal and deteriorated postural control systems.

$\dagger$ : Deteriorated postural control system is significantly different from normal one in both directions ( $\left.p_{\mathrm{AP}}=0.047, \mathrm{p}_{\mathrm{ML}}=\mathbf{0 . 0 0 0}\right)$

*: AP is significantly different from ML in normal postural control system $\left(p_{\text {Normal }}=0.000\right)$.

\section{(FIGURE 4)}




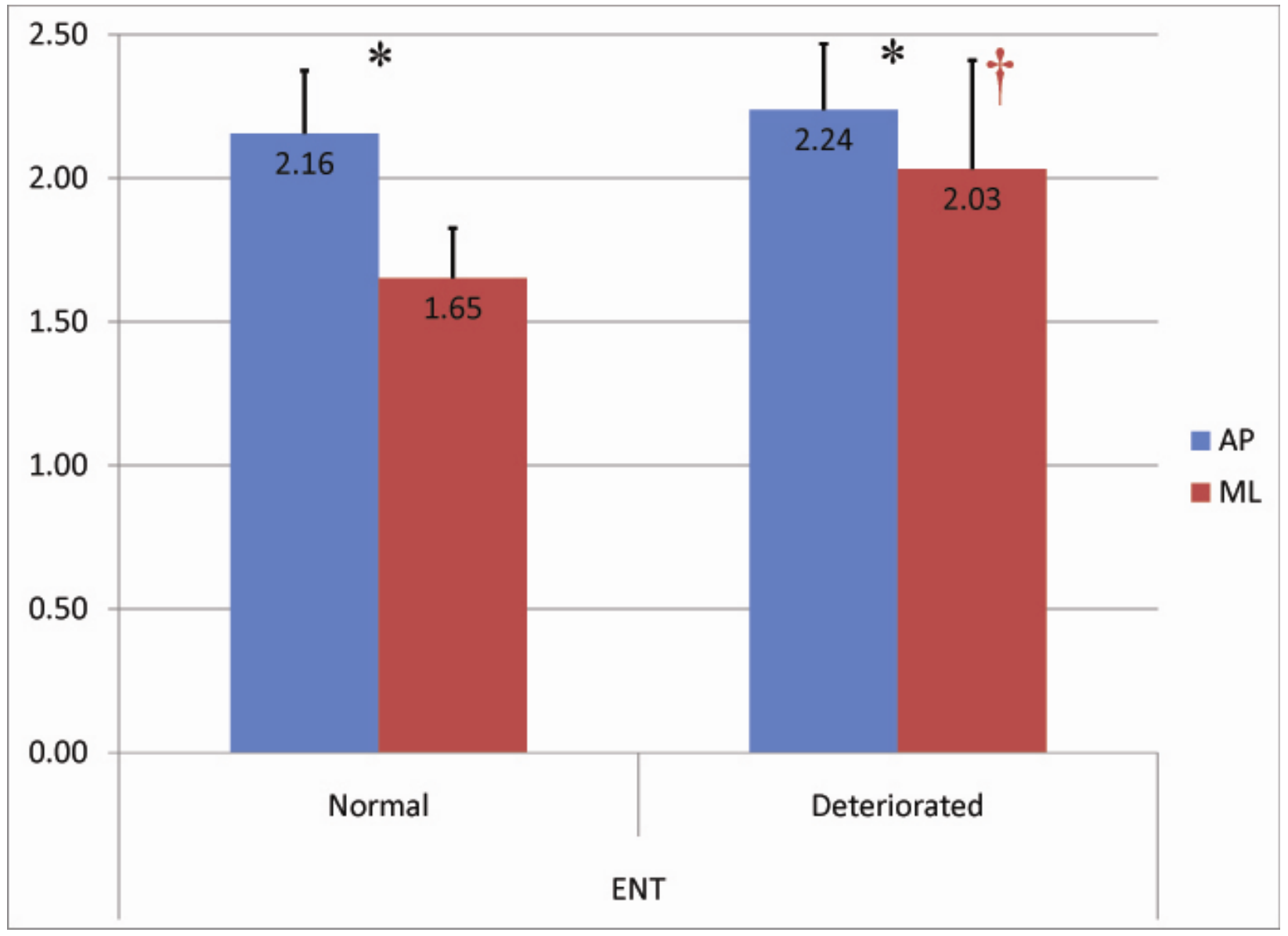

Fig 5. Entropy of embedded AP and ML COP time series of normal and deteriorated postural control systems.

$\dagger$ : Deteriorated postural control system is significantly different from normal one along ML direction $\left(\mathbf{p}_{M L}=\mathbf{0 . 0 0 0}\right)$.

*: AP is significantly different from $M L$ in both groups $\left(p_{\text {Normal }}=0.000, p_{\text {Deteriorated }}=0.002\right)$.

\section{(FIGURE 5)}




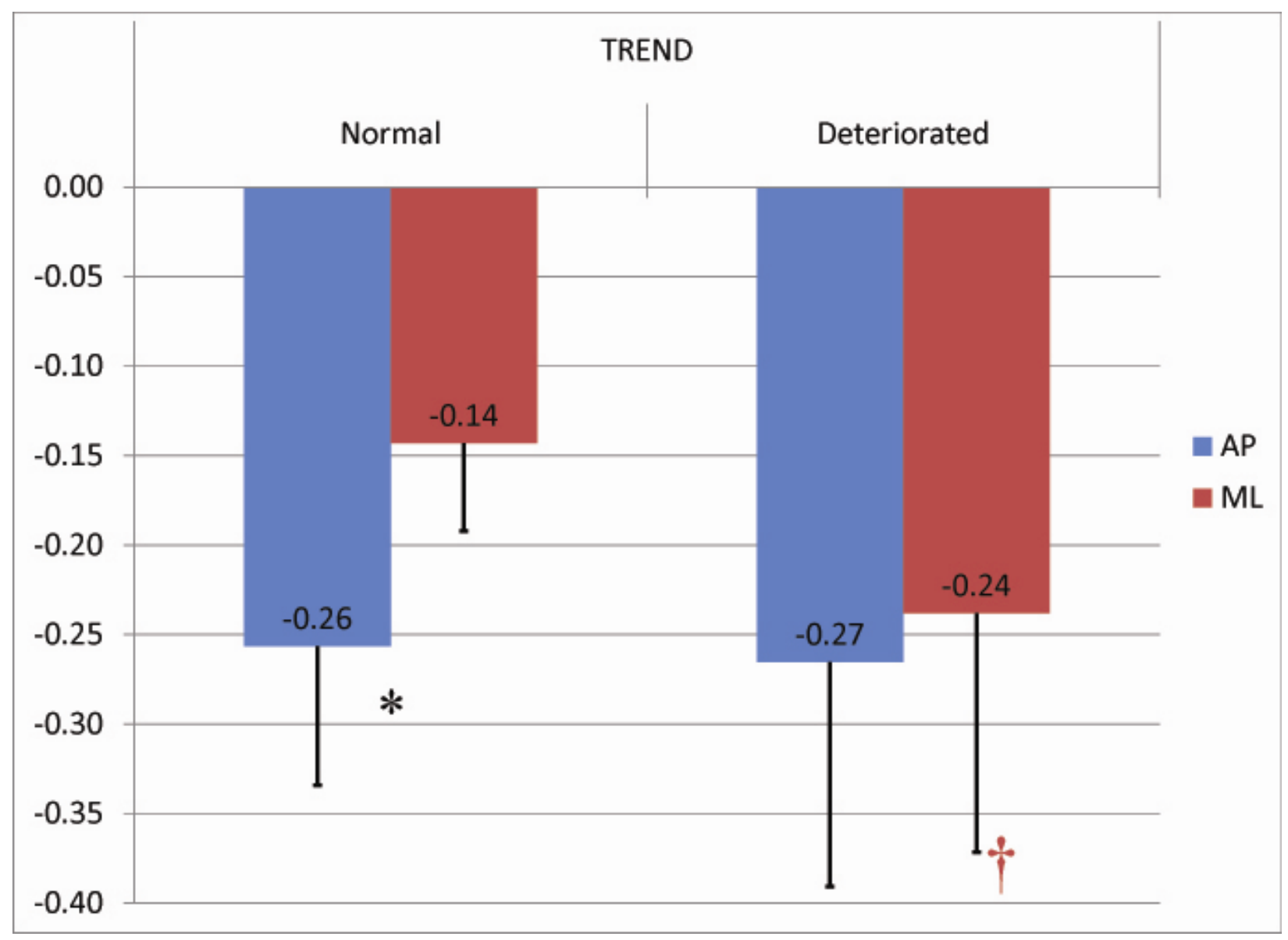

Fig 6. Trends of embedded AP and ML COP time series of normal and deteriorated postural control systems.

$\dagger^{\dagger}$ : Deteriorated postural control system is significantly different from normal one along ML direction ( $\left.\mathbf{p}_{M L}=\mathbf{0 . 0 0 2}\right)$.

*: AP is significantly different from ML in normal postural control system $\left(p_{\text {Normal }}=0.000\right)$.

\section{(FIGURE 6)}


Table 1. Descriptive statistics of first local minimums of auto mutual information functions of COP time series, embedding dimensions and mean distances between data points in reconstructed space.

\begin{tabular}{|c|c|c|c|c|}
\hline \multirow{2}{*}{ Group } & \multicolumn{2}{|c|}{ Normal } & \multicolumn{2}{c|}{ Deteriorated } \\
& \multicolumn{2}{|c|}{ Mean Value } & \multicolumn{2}{c|}{ Mean Value } \\
\cline { 2 - 5 } Index & AP & ML & AP & ML \\
\hline$\tau$ & 11 & 10 & 12 & 2 \\
\hline $\mathrm{d}$ & 2 & 2 & 2 & 1.5957 \\
\hline Mean & 1.6049 & 1.6049 & 1.6127 & \\
\hline Distance & & & & \\
\hline
\end{tabular}

\section{(TABLE 1)}

